\title{
EP-100
}

\section{Classification of Liver cirrhosis via data mining algorithm: A new tool to detect disease}

\author{
Manvendra SINGH*
}

Department of Computer Sciences, HMFA, Prayagraj, India

Introduction: Liver cirrhosis or hepatic cirrhosis is termed as condition in which the liver of patient not working properly owing to damage by various factors. In a present day, data mining in the field of medical is an important tool to explore the sign patterns in term of medical data sets. The aim of current study is to investigate a method for the classification system of liver cirrhosis in data findings in databases utilizing data mining method which are used in present time.

Methods: In the data mining system and research work, the Tanagra data mining device, an accurate classifier algorithm and an open source project is used. By using different classification algorithms, we find a pattern which is formed in it. Fischer filtering was good feature in feature selection algorithm. Limited number of attributes in filtering algorithm along with better classification system were chosen and executed. The architecture model flow includes: training dataset, feature relevance analysis, classification process i.e. classification and regression tree, random tree binary logistic regression, support vector machine accuracy analysis.

Results: The research work was accomplished on the basis classification algorithms including training and test datsets along with well obtained characterized data. The classification process categorized the liver cirrhosis in dataset and provides better results. The feature relevance analysis displays the different important characteristics which are intended for.

Conclusions: The experiment is projected to prove the efficiency of the presentation of numerous classifiers to the liver cirrhosis and dataset used in it. Primary diagnosis of any 